\title{
Thermosolutal convection in a rotating couple-stress fluid
}

\section{Lidia Palese}

Dipartimento di Matematica, Universit`a di Bari, Via E. Orabona 4, 70125 Bari, Italy

\section{Abstract:}

In this paper we study the nonlinear Lyapunov stability of the conduction-diffusion solution of a rotating couple-stress fluid, in a layer heated and salted from below.

After reformulating the perturbation evolution equations in a suitable equivalent form, we derive the appropriate Lyapunov function and we prove that, if the principle of exchange of stabilities holds, the linear and nonlinear stability bounds are equal.

The nonlinear stability bound is exactly the critical Rayleigh number obtained solving the linear instability problem of the conduction-diffusion solution.

Key words: Stability - Bénard problem - Energy Method.

MSC 76E15-76E30

\section{Council for Innovative Research}

Peer Review Research Publishing System

Journal: JOURNAL OF ADVANCES IN MATHEMATICS

Vol .11, No. 5

www.cirjam.com , editorjam@gmail.com 


\section{INTRODUCTION}

The problem of thermal convection in a fluid layer, i.e. the classical Bénard problem, of a big importance in astrophisics, geophysics, oceanography, meteorology, has been largely investigated [1], [2], [3], [4], [5], in the Oberbeck- Boussinesq approximation [3]-[12] too, including the influence, on the onset on thermal instability, of effects such as a rotation field, a magnetic field, chemical reactions of reactive fluids.

In [11], [12] the problem of the coincidence of the critical and nonlinear stability bounds was studied, and in [11] the coincidence of linear and nonlinear stability parameters was deduced, under some restriction on initial data.

In the magnetohydrodynamic case, [13], for free boundaries, the coincidence of linear and nonlinear stability parameters was deduced for a fully ionized fluid, i.e., if the conduction diffusion solution is linearly stable, it is conditionally nonlinearly asymptotically stable.

In [14],[15] and [16] the hydrodynamic stability of the thermodiffusive equilibrium was investigated for a Newtonian fluid mixture in a horizontal layer heated from below and the thermoanisotropic effects on the hydrodynamic stability of the mechanical equilibrium have been evaluated.

The given problem has been changed in an equivalent one, with better symmetry properties, a system equivalent to the perturbation evolution equations is derived [17]

For reactive fluids of technological interest, chemical reactions [5] can give temperature and concentration gradients which can alter hydrodynamic stabilities.

A nonlinear stability analysis of the conduction-diffusion solution of the $\mathrm{Be}^{\prime}$ nard problem, in presence of chemical surface reactions was performed in [18], [19] and [20], showing, in the case of coincidence of Prandtl and Schmidt numbers, the equality between linear and nonlinear stability bounds.

The nonlinear stability of the thermodiffusive equilibrium for a homogeneous fluid in a horizontal rotating layer with free boundaries was considered in [21], i.e. the rotating Bénard problem .

The mathematical problem of the nonlinear Lyapunov stability was reformulated, obtaining a system of perturbation evolution equations in some suitable orthogonal subspaces, preserving the contribution of the skewsymmetric rotation term.

If the principle of exchange of stabilities holds, the equality between linear and nonlinear stability bounds was obtained.

In [22] was reformulated, in a simpler way, the nonlinear stability problem. The contribution of a skewsymmetric linear term, such as the Coriolis term in the rotating Bénard problem [23], can be preserved also by using the classical $L^{2}$ norm for the perturbation energy, but only projecting the initial perturbation equations on suitable orthogonal subspaces, after reformulating the mathematical stability problem.

For a viscous, non Newtonian fluid, such as a couple-stress fluid,whose theory has been formulated by Stokes in 1966 [24], the thermosolutal convection is a problem of a big importance in several fields of fluid and magnetofluid mechanics.

A remarkable application is given by the human joints, where articular cartilage acts as bearing and the sinovial fluid as a lubrifiant. The sinovial fluid, a non Newtonian viscous fluid, according to the Stokes theory, has been modeled as a couple-stress fluid [25], since the hyaluronic acid mulecules are very large mulecules that can be found as additives in sinovial fluids.

A lot of theoretical results on couple-stress fluids, taking into account several effects, can be found in [26], where, in the framework of the linearized stability theory, by using the normal modes technique, the thermosolutal convection in a layer of electrically couple stress conducting fluid was considered. In the case of stationary convection a linear instability analysis was performed, obtaining the dispersion relation, also numerically analized.

In this paper we consider a couple stress fluid heated and salted from below, in a horizontal rotating layer with free boundaries, and we study the nonlinear stability of the thermodiffusive equilibrium.

We consider the initial-boundary value problem for the perturbation fields in Section 2, in Section 3 we formulate the mathematical problem in an equivalent form, in some suitable orthogonal Sobolev spaces, in Section 4 we study the Lyapunov stability, [14], [22], on a suitable linear space of admissible vector functions and, we derive a quadratic function $E_{L}$.

The inequality $\frac{d E_{L}}{d t} \leq 0$, when $E_{L}$ is a Lyapunov function, represents a sufficient condition for global nonlinear Lyapunov stability.

Solving the Euler equations associated with the resulting maximum problem, in Section 5, we determine a sufficient condition of global nonlinear Lyapunov stability.

\section{The initial/boundary value problem for perturbation}


In the framework of physics of continua, let us consider a couple-stress fluid, subject to the gravity field $\vec{g}$, in a horizontal layer $S$ bounded by the surfaces $z=0$ and $z=d$ in a frame of reference $\{O, \vec{i}, \vec{j}, \vec{k}\}$, with $\vec{k}$ unit vector in the vertical upwards direction.

We assume the fluid, heated and salted from below, subject to some constant vertical temperature and concentration gradients $\beta$ and $\gamma$, in rotation around the fixed vertical axis $z$ with a constant angular velocity $\vec{\Omega}=\Omega \vec{k}$.

The motion which occurs in $S$, for an observer rotating around the same axis $z$ with the same angular velocity $\vec{\Omega}$, in the Oberbeck-Boussinesq approximation, is described by the following equations [1], [24], [26]

$$
\frac{\partial}{\partial t} \vec{v}+(\vec{v} \cdot \nabla) \vec{v}=-\frac{\nabla p}{\rho_{0}}+\left[1-\alpha\left(T-T_{0}\right)+\alpha^{\prime}\left(C-C_{0}\right)\right] \vec{g}+\vec{v} \times \vec{\Omega}+v \Delta \vec{v}-v^{\prime} \Delta \Delta \vec{v},
$$

$$
\begin{gathered}
\frac{\partial}{\partial t} T+\vec{v} \cdot \nabla T=K_{T} \Delta T, \\
\frac{\partial}{\partial t} C+\vec{v} \cdot \nabla C=K_{C} \Delta C, \\
\nabla \cdot \vec{v}=0,
\end{gathered}
$$

where $\vec{v}, T, C, p$ are the velocity, temperature, solute concentration and pressure fields. $\rho_{0}, \alpha, \alpha^{\prime}, v, v^{\prime}, K_{T}$ and $K_{C}$ are positive constant which represent the density of the fluid at a reference temperature, the thermal coefficient of the volume expansion, the solute coefficient of the volume expansion, the kinematic viscosity, the couple-stress viscosity, the thermal conductivity, and the solute conductivity, respectively. $T_{0}$ and $C_{0}$ are a constant reference temperature and solute concentration .

$\nabla$ and $\Delta$ stand for gradient and Laplacian operators.

We assume the boundaries of the layer $S$ stress free and conductors of heat and solute concentration. In this case the boundary conditions read [1]

$$
\left\{\begin{aligned}
\vec{v} \cdot \vec{n}=\vec{n} \times \vec{D} \cdot \vec{n}=\overrightarrow{0}, & & z=0, d & \\
T=T^{0}, & & C=C^{0} \quad z=0, & t \geq 0 \\
T=T^{1}, & & C=C^{1} \quad z=d, & t \geq 0
\end{aligned}\right.
$$

where $\vec{D}$ is the stress tensor, $\vec{n}$ is the external normal to the layer boundary and $T^{0}, T^{1}$ and $C^{0}, C^{1}$ are, respectively, prescribed temperatures and conentrations on the walls of the layer.

Let us now perturb the zero solution corresponding to a motionless state,

$$
\left\{\vec{v}=\overrightarrow{0}, \bar{T}=-\beta z+T^{0}, \bar{C}=-\gamma z+C^{0}, \bar{P}=\bar{P}(z)\right\},
$$

$\beta=\frac{T^{0}-T^{1}}{d}, \gamma=\frac{C^{0}-C^{1}}{d}$, up to a cellular motion characterized by a velocity $\vec{u}=\overrightarrow{0}+\vec{u}$, a pressure $p=\vec{P}+p^{\prime}$, a temperature $T=\bar{T}+\theta$, a solute concentration $C=\bar{C}+\gamma$.

The perturbation fields $\vec{u}, p^{\prime}, \theta, \gamma$ satisfy the following nondimensional equations

$$
\frac{\partial}{\partial t} \vec{u}+(\vec{u} \cdot \nabla) \vec{u}=-\nabla p^{\prime}+R \theta \vec{k}-R_{c} \vec{\jmath}+\vec{u} \times \vec{\Omega}+\Delta \vec{u}-F \Delta \Delta \vec{u},
$$




$$
\begin{gathered}
P_{r}\left(\frac{\partial}{\partial t} \theta+\vec{u} \cdot \nabla \theta\right)=\Delta \theta+R w, \quad(t, \vec{x}) \in(0, \infty) \times V \\
S_{c}\left(\frac{\partial}{\partial t} \gamma+\vec{u} \cdot \nabla \gamma\right)=\Delta \gamma-R_{c} w, \quad(t, \vec{x}) \in(0, \infty) \times V \\
\nabla \cdot \vec{u}=0,
\end{gathered}
$$

in the following subset of the Sobolev space $W^{2,2}(V)$,

$$
N=\left\{(\vec{u}, p, \theta,) \in W^{2,2}(V) \mid \frac{\partial}{\partial z} u=\frac{\partial}{\partial z} v=w=\theta=\gamma=0 \mathrm{o} n \partial V\right\},
$$

where $\vec{u}=(u, v, w)$, and $V=V \times[0,1]$ denotes the three dimensional box over the rectangle $V$, periodic in the $x, y$ directions and its boundary is denoted by $\partial V$, after assuming the perturbation fields, depending on the time $t$ and space $\vec{x}=(x, y, z)$, doubly periodic functions in $x$ and $y$, of period $2 \pi k_{1}$ and $2 \pi k_{2}$.

$R^{2}, R_{c}^{2}$, are the Rayleigh and solute Rayleigh numbers, $P_{r}$ and $S_{c}$ are the Prandtl and Schmidt numbers, respectively. $F$ is a dimensionless couple-stress parameter.

\section{The evolution equations for the perturbation fields}

If the mean values of $u, v, w$ vanish over $V$ [3], [27], that is if the conditions

$$
\int_{V} u(x, y, z) d x d y=\int_{V} v(x, y, z) d x d y=\int_{V} w(x, y, z) d x d y=0, \quad z \in[0,1],
$$

hold, the velocity perturbation $\vec{u}$ has the unique decomposition [3]

$$
\vec{u}=\vec{u}_{1}+\vec{u}_{2}
$$

with

$$
\begin{gathered}
\nabla \cdot \vec{u}_{1}=\nabla \cdot \vec{u}_{2}=\vec{k} \cdot \nabla \times \vec{u}_{1}=\vec{k} \cdot \vec{u}_{2}=0, \\
\vec{u}_{1}=\nabla \frac{\partial}{\partial z} \chi-\vec{k} \Delta \chi \equiv \nabla \times \nabla \times(\chi \vec{k}), \\
\vec{u}_{2}=\vec{k} \times \nabla \psi=-\nabla \times(\vec{k} \psi),
\end{gathered}
$$

where the poloidal and toroidal potentials $\chi$ and $\psi$ are doubly periodic satisfying the equations [3]

$$
\Delta_{1} \chi \equiv \frac{\partial^{2}}{\partial x^{2}} \chi+\frac{\partial^{2}}{\partial y^{2}} \chi=-\vec{k} \vec{u}, \quad \Delta_{1} \psi=\vec{k} \cdot \nabla \times \vec{u} .
$$

The boundary conditions for $\chi$ and $\psi$, for free planar surfaces, are [3]:

$$
\chi=\chi_{z z}=\psi_{z}=0 \quad z=0,1 .
$$

From (12)-(13) it follows that

$$
\vec{u} \cdot \vec{k}=\vec{u}_{1} \cdot \vec{k}=-\Delta_{1} \chi,
$$

where $\Delta_{1}$ stands for the twodimensional Laplace operator.

In order to project the perturbation equation (7) on some suitable subspaces of $L^{2}(V)$ we observe that

$$
\vec{u}=\nabla \times \nabla \times(\chi \vec{k})-\nabla \times(\psi \vec{k}),
$$

because of $\nabla \cdot \vec{u}=0$,

$$
\Delta \vec{u}=-\nabla \times \nabla \times \vec{u}
$$


Let us recall the Weyl decomposition theorem [4], [29]

$$
L^{2}(V)=G(V) \oplus N(V)
$$

with $G(V)$ and $N(V)$ spaces of generalized solenoidal and potential vectors respectively. So, the advective term in (7) can be uniquely obtained as

$$
(\vec{u} \cdot \nabla)\left(\vec{u}_{1}+\vec{u}_{2}\right)=\nabla U+\nabla \times \vec{A}
$$

where $U$ is a scalar function and $\vec{A}$ a vector field we specify later.

The scalar function $U$ is (up to a constant) the solution of the interior Neumann problem [29] in the periodicity cell $V$ [21]-[23]

$$
\begin{gathered}
\Delta U=\Phi \\
\frac{\partial}{\partial \vec{n}} U=\Gamma,
\end{gathered}
$$

where $\frac{\partial}{\partial \vec{n}} U$ is the normal derivative of $U$ on the boundary $\partial V$ of the periodicity cell $V$, and $\Gamma=-\vec{B} \cdot \vec{n}$.

It is well known that the interior Newmann problem in the general case has no solution, only if the relation

$$
\int_{V} \Phi d v-\int_{\partial V} \Gamma d v=\int_{\partial V}\left(\vec{u} \cdot \nabla\left(\vec{u}_{1}+\vec{u}_{2}\right)\right) \cdot \vec{n} d \sigma+\int_{V} \nabla \cdot \vec{B} d v=0
$$

is fulfilled, a solution of (23), (24) can exist.

The perturbation equation (7), taking into account (19), (20), (21), becomes [22]:

$$
\begin{gathered}
\frac{\partial}{\partial t}(\nabla \times \nabla \times(\chi \vec{k})-\nabla \times(\psi \vec{k}))+\nabla U+\nabla \times \vec{A}=-\nabla p^{\prime}+\nabla_{1}(\Omega \psi)- \\
-\nabla \times(\vec{\Omega} \times \nabla \times(\chi \vec{k}))-\nabla \times \nabla \times(\nabla \times \nabla \times(\chi \vec{k})-\nabla \times(\psi \vec{k}))- \\
F \nabla \times \nabla \times \nabla \times \nabla \times(\nabla \times \nabla \times(\chi \vec{k})-\nabla \times(\psi \vec{k}))+ \\
+P\left(R \theta \vec{k}-R_{c} \vec{k}\right)+(I-P)\left(R \theta \vec{k}-R_{c} \vec{k}\right)
\end{gathered}
$$

where $I$ and $P$ are, respectively, the identity operator and the projection on the subspace $G(V)$ of $L^{2}(V)$. After assuming that every term of the equation (26), as a function of the space variable $\vec{x}$, belongs to the Sobolev space $W^{2,2}(V)$, if we project (26) on the subspace of solenoidal and potential vectors, taking into account that the only vector belonging to both previous subspaces is null [4], from the imbedding Sobolev theorems of $W^{2,2}(V)$ in the space of continuous functions $C(\bar{V})$ [28], it follows that

$$
\nabla U=-\nabla p^{\prime}+\nabla_{1}(\psi \Omega)++P\left(R \theta \vec{k}-R_{c} \vec{k}\right)
$$

If we consider the scalar product of $(27)$ by $\vec{u}_{1}$, which is solenoidal, we obtain

$$
\left(\nabla_{1}(\psi \Omega), \vec{u}_{1}\right)=0
$$

that, in terms of poloidal and toroidal fields becomes

$$
\left(\psi_{x}, \chi_{x z}\right)+\left(\psi_{y}, \chi_{y z}\right)=0
$$

This is the equation that allows us to preserve the contribution of the skewsymmetric term in nonlinear Lyapunov stability, as we shall see later.

\section{Lyapunov stability}


If we multiply (7) by $\vec{u},(8)$ by $\frac{b}{P_{r}} \theta$ and (9) by $\frac{d}{S_{c}} \gamma$, where $b, d$ are some positive parameter, adding the resulted equations and integrating over $V$, we obtain

$$
\begin{gathered}
\frac{d}{d t} \frac{1}{2} \int_{V}\left\{\left(\vec{u}^{2}+b \theta^{2}+d \gamma^{2}\right\} d V=R\left(1+\frac{b}{P_{r}}\right)(\theta, w)-R_{c}\left(1+\frac{d}{S_{c}}\right)(\gamma, w)+\right. \\
+\frac{b}{P_{r}}(\theta, \Delta \theta)+\frac{d}{S_{c}}(\gamma, \Delta \gamma)+(\vec{u}, \Delta \vec{u})-F(\vec{u}, \Delta \vec{u}) .
\end{gathered}
$$

Let us introduce the function

$$
E_{L}(t)=\frac{1}{2}\left\{\left|\chi_{x z}\right|^{2}+\left|\chi_{y z}\right|^{2}+\left|\Delta_{1} \chi\right|^{2}+\left|\psi_{y}\right|^{2}+\left|\psi_{x}\right|^{2}+b|\theta|^{2}+d|\gamma|^{2}\right\}
$$

Taking into account (29), (30) and (31), we can write again the previous energy relation as follows

$$
\frac{d}{d t} E_{L}=R I-E
$$

where

$$
\begin{gathered}
I \equiv-\left(1+\frac{b}{P_{r}}\right)\left(\theta, \Delta_{1} \chi\right)+\frac{R_{c}}{R}\left(1+\frac{d}{S_{c}}\right)\left(\gamma, \Delta_{1} \chi\right)+\frac{f(\vec{\Omega})}{R}\left(\left(\psi_{x}, \chi_{x z}\right)+\left(\psi_{y}, \chi_{y z}\right)\right)+ \\
F\left[\left(\chi, \Delta_{1} \chi_{z z z z}\right)-\left(\chi_{z z}, \Delta_{1} \chi_{z z}\right)-\left(\psi, \Delta_{1} \psi_{z z}\right)-\left(\psi_{z}, \Delta_{1} \psi_{z}\right)\right] \\
E \equiv \frac{b}{P_{r}}|\nabla \theta|^{2}+\frac{d}{S_{c}}|\nabla \gamma|^{2}+\left[\left|\nabla \chi_{x z}\right|^{2}+\left|\nabla \chi_{y z}\right|^{2}+\left|\nabla \Delta_{1} \chi\right|^{2}\right]+ \\
\quad+F\left[\left|\nabla \nabla \chi_{x z}\right|^{2}+\left|\nabla \nabla \chi_{y z}\right|^{2}+\left|\nabla \nabla \Delta_{1} \chi\right|^{2}\right]+ \\
+\left[\left|\nabla \psi_{x}\right|^{2}+\left|\nabla \psi_{y}\right|^{2}+F\left(\left|\nabla \nabla \psi_{x}\right|^{2}+\left|\nabla \nabla \psi_{y}\right|^{2}\right)\right]
\end{gathered}
$$

in (33) $f(\Omega)$ is an arbitrary function of $\Omega$, we determine later.

We determine a condition ensuring that

$$
\frac{d}{d t} E_{L} \leq 0, \quad \forall t \geq 0
$$

The equation (32) becomes:

$$
\frac{d}{d t} E_{L}=R I-E=-E\left(1-R \frac{I}{E}\right) .
$$

If

$$
R<\sqrt{R a_{*}}
$$

where

$$
\frac{1}{\sqrt{R_{a^{*}}}}=\max \frac{I}{E}
$$

in the class of admissible functions satisfying the boundary conditions (11), from (37), (38) and (39) we deduce

$$
\frac{d}{d t} E_{L} \leq-\left(1-\frac{R}{\sqrt{R_{a^{*}}}}\right) E .
$$


Hence, if (38) is satisfied, the functional $E_{L}$ is a decreasing function of $t$. The inequality (36) represents a stability uniqueness criterion [3], [4].

We observe explicitely that some Sobolev imbedding theorems, Schwarz, Wirtinger and Poincaré inequalities allow us to prove the boundedness of the functional $\frac{I}{E}$ in the class of admissible functions satisfying the boundary conditions (11).

\section{The maximum problem and the stability bound}

We will study the variational problem (39) and later determine the parameters $b, d$ in terms of the physical quantities, such that $\sqrt{R_{a^{*}}}$ will be maximal.

The Euler Lagrange equations associated with the maximum problem (39) are:

$$
\begin{gathered}
-\left(1+\frac{b}{P_{r}}\right) \Delta_{1} \theta+\frac{R_{c}}{R}\left(1+\frac{d}{S_{c}}\right) \Delta_{1} \gamma+\frac{f(\vec{\Omega})}{R} \Delta_{1} \psi_{z}+\frac{2}{\sqrt{R_{a^{*}}}}\left[\Delta \Delta_{1} \chi-F \Delta \Delta \Delta_{1} \chi\right]=0, \\
-\left(1+\frac{b}{P_{r}}\right) \Delta_{1} \chi+\frac{b}{P_{r}} \frac{2}{\sqrt{R_{a^{*}}}} \Delta \theta=0, \\
\frac{R_{c}}{R}\left(1+\frac{d}{S_{c}}\right) \Delta_{1} \chi+\frac{d}{S_{c}} \frac{2}{\sqrt{R_{a^{*}}}} \Delta \gamma=0, \\
\frac{f(\vec{\Omega})}{R} \Delta_{1} \chi_{z}+\frac{2}{\sqrt{R_{a^{*}}}}\left[\Delta_{1} \psi-F \Delta_{1} \psi\right]=0 .
\end{gathered}
$$

Taking into account (16), the system of Euler equations equivalently read

$$
\begin{gathered}
-\left(1+\frac{b}{P_{r}}\right) \Delta_{1} \theta+\frac{R_{c}}{R}\left(1+\frac{d}{S_{c}}\right) \Delta_{1} \gamma+\frac{f(\vec{\Omega})}{R} \zeta_{z}-\frac{2}{\sqrt{R_{a^{*}}}}(\Delta \Delta w-F \Delta \Delta \Delta w)=0, \\
\left(1+\frac{b}{P_{r}}\right) w+\frac{b}{P_{r}} \frac{2}{\sqrt{R_{a^{*}}}} \Delta \theta=0, \\
-\frac{R_{c}}{R}\left(1+\frac{d}{S_{c}}\right) w+\frac{d}{S_{c}} \frac{2}{\sqrt{R_{a^{*}}}} \Delta \theta=0, \\
-\frac{f(\vec{\Omega})}{R} w_{z}+\frac{2}{\sqrt{R_{a^{*}}}}[\Delta \zeta-F \Delta \Delta \zeta]=0,
\end{gathered}
$$

where $\zeta \equiv \Delta_{1} \psi$

We shall assume that the principle of exchange of stabilities holds, i.e. the instability occurs as a stationary convection. In the class of normal mode perturbations

$$
\begin{aligned}
& w(\vec{x})=W(z) \exp \left[i\left(k_{1} x_{1}+k_{2} x_{2}\right)\right] \\
& \zeta(\vec{x})=Z(z) \exp \left[i\left(k_{1} x_{1}+k_{2} x_{2}\right)\right] \\
& \theta(\vec{x})=\Theta(z) \exp \left[i\left(k_{1} x_{1}+k_{2} x_{2}\right)\right], \\
& \gamma(\vec{x})=\Gamma(z) \exp \left[i\left(k_{1} x_{1}+k_{2} x_{2}\right)\right],
\end{aligned}
$$

the equations (43) become 


$$
\begin{gathered}
k^{2}\left(1+\frac{b}{P_{r}}\right) \Theta-\frac{R_{c}}{R} k^{2}\left(1+\frac{d}{S_{c}}\right) \Gamma+\frac{f(\vec{\Omega})}{R} D Z-\frac{2}{\sqrt{R_{a^{*}}}}\left[\left(D^{2}-k^{2}\right)^{2}-F\left[\left(D^{2}-k^{2}\right)^{3}\right] W=0,\right. \\
\left(1+\frac{b}{P_{r}}\right) W+\frac{b}{P_{r}} \frac{2}{\sqrt{R_{a^{*}}}}\left(D^{2}-k^{2}\right) \Theta=0, \\
-\frac{R_{c}}{R}\left(1+\frac{d}{S_{c}}\right) W+\frac{d}{S_{c}} \frac{2}{\sqrt{R_{a^{*}}}}\left(D^{2}-k^{2}\right) \Gamma=0, \\
-\frac{f(\vec{\Omega})}{R} D W+\frac{2}{\sqrt{R_{a^{*}}}}\left[\left(D^{2}-k^{2}\right)-F\left[\left(D^{2}-k^{2}\right)^{2}\right] Z=0,\right.
\end{gathered}
$$

where $k^{2}=k_{1}^{2}+k_{2}^{2}$ is the wave number.

To (45) we add the following boundary conditions:

$$
W=D^{2} W=\Theta=D^{2} \Theta=\Gamma=D^{2} \Gamma=D Z=0 .
$$

Owing to (16), and (17), we assume [5]

$$
W(z)=\sum_{n=1}^{\infty} W_{n} \sin (n \pi z)
$$

From (43)and (47) we have

$$
\begin{gathered}
R_{a^{*}}\left(R^{2}, R_{c}^{2}, F, k^{2}, n^{2} \pi^{2}, b, d\right)= \\
\frac{4 R^{2}\left(n^{2} \pi^{2}+k^{2}\right)\left[\left(n^{2} \pi^{2}+k^{2}\right)^{2}+F\left(n^{2} \pi^{2}+k^{2}\right)^{3}\right]}{\frac{-[f(\vec{\Omega})]^{2} n^{2} \pi^{2}}{1+F\left(n^{2} \pi^{2}+k^{2}\right)}+k^{2} R^{2} \frac{P_{r}}{b}\left(1+\frac{b}{P_{r}}\right)^{2}-k^{2} R_{c}^{2} \frac{S_{c}}{d}\left(1+\frac{d}{S_{c}}\right)^{2}} .
\end{gathered}
$$

defined on the subset of the parameter space where the denominator is definite positive.

Differentiating (48) with respect to the parameters $b$, and $d$ we obtain

$$
\frac{\partial}{\partial b} R_{a^{*}}=0 \Leftrightarrow \frac{b}{P_{r}}=1, \quad \frac{\partial}{\partial d} R_{a^{*}}=0 \Leftrightarrow \frac{d}{S_{c}}=1 .
$$

Substituting (49) in (48) we obtain $R_{a^{*}}$ as a function of $R^{2}$ and $R_{c}^{2}$

$$
R_{a^{*}}\left(R^{2}, R_{c}^{2}, F, k^{2}, n^{2} \pi^{2}\right)=\frac{R^{2}\left(n^{2} \pi^{2}+k^{2}\right)^{3}\left[1+F\left(n^{2} \pi^{2}+k^{2}\right)\right]}{\frac{-[f(\vec{\Omega})]^{2} n^{2} \pi^{2}}{4\left[1+F\left(n^{2} \pi^{2}+k^{2}\right)\right]}+k^{2} R^{2}-k^{2} R_{c}^{2}},
$$

defined on the subset $\frac{-[f(\vec{\Omega})]^{2} n^{2} \pi^{2} c^{2}}{4\left[1+F\left(n^{2} \pi^{2}+k^{2}\right)\right]}+k^{2} R^{2}-k^{2} R_{c}^{2}>0$.

The critical Rayleigh function of the linear instability theory, that is

$$
R_{c r}^{2}=R_{c}^{2}+\frac{\left(n^{2} \pi^{2}+k^{2}\right)^{3}\left[1+F\left(n^{2} \pi^{2}+k^{2}\right)\right]^{2}+4 \Omega^{2} n^{2} \pi^{2}}{k^{2}\left[1+F\left(n^{2} \pi^{2}+k^{2}\right)\right]},
$$

belongs to the subset where the denominator of $(50)$ is definite positive, choosing $f(\Omega)=4 \Omega$. 
Evaluating (50) for $R^{2}=R_{c r}^{2}$, we obtain

$$
R_{c r}^{2}\left(k^{2}, n^{2} \pi^{2}\right)=R_{a^{*}}\left(k^{2}, n^{2} \pi^{2}\right) .
$$

Hence we proved the following theorem

Theorem 5.1 If the principle of exchange of stabilities holds, the zero solution of (7)-(11), corresponding to the basic conduction state is nonlinearly globally stable if

$$
R^{2}<R_{a^{*}}
$$

where $R_{c r}^{2}=R_{a^{*}}\left(R_{c r}^{2}, F, k^{2}, n^{2}\right)$, attains its minimum where $n=1$. Whence the linear and non linear stability bounds, obtained for $n=1$

$$
R_{c r}^{2}\left(k^{2}, F\right)=R_{a^{*}}\left(R_{c r}^{2}, k^{2}, F\right)
$$

coincide.

\section{Conclusions}

We studied the nonlinear stability of the motionless state for a couple-stress fluid in a rotating horizontal layer, heated and salted from below, considering the proiection of perturbation evolution equations, written in terms of in terms of toroidal and poloidal fields, in some orthogonal subspaces of $L^{2}(V)$.

In this way, we obtain an energy relation for the Lyapunov function in which all the nonlinear terms disappear and the skewsymmetric rotation term is preserved.

We studied the nonlinear Lyapunov stability and determine a sufficient condition for global stability.

After solving, in the class of normal modes, the Euler-Lagrange equations associated with the maximum problem, , we maximize the stability domain with respect to the parameters introduced in the Lyapunov functional and we deduce if the principle of exchange of stabilities holds, the equality between the linear and nonlinear critical parameters for the global stability.

We observe that, anyhow, in this paper we applied an idea similar to [14] [15] [16] [18] [19], where, we modified the evolution equations obtaining equations with better symmetries, which incorporate the given equations. In this way, [14], [22], [23] , we preserve the contribution of some skewsymmetric terms, otherwise, if the initial evolution equations were used such contribution was null and, correspondingly, the stability criterion, weaker.

The given problem governing the perturbation evolution was changed in order to obtain an optimum energy relation, whence a linearization principle in the sense of the coincidence of both linear and nonlinear stability bounds.

Whence we determine a sufficient condition of nonlinear stability that is also a necessary and sufficient condition of linear stability

\section{References}

[1] S.Chandrasekhar,Hydrodynamic and Hydromagnetic stability, Oxford, Clarendon Press, 1968.

[2] D.D. Joseph, Global Stability of the Conduction-Diffusion Solution, Arch. Rat. Mech. Anal., 36, 4 (1970), $285-292$.

[3] D.D. Joseph, Stability of fluid motions I-II, Springer, Berlin, 1976.

[4] A. Georgescu, Hydrodynamic stability theory, Kluwer, Dordrecht, 1985.

[5] B. Straughan, The Energy Method, Stability and Nonlinear Convection, 2nd. ed. Ser. in Appl. Math. Sci., 91, Springer, New York, 2003.

[6] G. P. Galdi, M. Padula, A new approach to energy theory in the stability of fluid motion, Arch. Rat. Mech. Anal., 110, 3, (1990), 187-286.

[7] G. P. Galdi, B. Straughan, A nonlinear analysis of the stabilizing effect of rotation in the Bénard problem., Proc. Royal Soc. of London, Ser. A, 402 (1823), (1985), 257-283.

[8] S. Rionero, Sulla stabilit $a$ magnetoidrodinamica in media con vari tipi di condizioni al contorno, Ricerche di Mat., 17, 4 (1968), 64-78.

[9] S. Rionero, G. Mulone, On the nonlinear stability of a thermodiffusive fluid mixture in a mixed problem, J. Math. Anal. Appl., 124, (1987), 165-188. 
[10] S. Rionero, G. Mulone, A nonlinear stability analysis of the magnetic $\mathrm{B} e^{\prime}$ nard problem through the Liapunov direct method,, Arch.Rat. Mech. Anal., 103, 4 (1988), 347-368.

[11] G. Mulone, S. Rionero, The rotating Bénard problem: new stability results for any Prandtl and Taylor numbers, Continuum Mech. Thermody., 9, (1997), 347-363.

[12] G. Mulone, Stabilizing effects in dynamical systems: linear and nonlinear stability conditions, Far East J. Appl. Math., 15, 2, (2004), 117-134.

[13] L. Palese, On the nonlinear stability of the MHD anisotropic Bénard problem, Int. J. Engng. Sc., 43, (2005), 12651282.

[14] A. Georgescu, L. Palese, Extension of a Joseph's criterion to the non linear stability of mechanical equilibria in the presence of thermodiffusive conductivity, Theor. and Comp. Fluid Mech., 8, 1996, 403-413.

[15] A. Georgescu, L. Palese, A. Redaelli, On a New Method inHydrodynamic Stability Theory, Math. Sc. Res. Hot Line, 4, 7 (2000), 1-16.

[16] A. Georgescu, L. Palese, A. Redaelli, The Complete Form for the Joseph Extended Criterion, Annali Univ. Ferrara , Sez. VII, Sc. Mat. 48, (2001), 9-22.

[17] A. Georgescu, L. Palese, Stability Criteria for Fluid Flows, Advances in Math. for Appl. Sc., 81, World Scient. Singapore, 2010.

[18] A. Georgescu, L. Palese, On the nonlinear stability of a binary mixture with chemical surface reactions, Annals of the Academy of Romanian Scientists, Series on Math. and Appl. 3, 1, (2011), 106-115.

[19] A. Georgescu, L. Palese, A linearization principle for the stability of the chemical equilibrium of a binary mixture Romai Journal, 6, 2 (2010), 131-138.

[20] L. Palese, On the nonlinear stability of a binary mixture with chemical surface reactions in the general case , Electronic Journal Differential Equations, 2014, 187, (2014), 1-12.

[21] L. Palese, On the stability of the rotating Bénard problem . Annals of the Academy of Romanian Scientists, Series on Math. and Appl., 1 (2014), 4-18.

[22] L. Palese, On the Lyapunov function for some convection problems in hydrodynamic . Boll. mat. Pura appl. ( 2014).

[23] L. Palese, On the Lyapunov function for the rotating benard problem . Journal of Advances in Mathematics, 9, 9 , (2015) 3063-3072..

[24] V. K. Stokes, Couple stresses in fluids, Phys. of Fluids, 9, 1966, 1709-1715.

[25] E. Walicki, A. Walicka, Inerzia effects in the squeeze film of a couple stress fluid in biological bearing, Appl. Mech. Engng. 4, 1999, 363-373.

[26] P. Kumar, Thermosolutal Magneto-Rotatory Convection In Couple - Stress Fluid, J. of Appl. Fluid Mech., 5, 4, (2012), 45-52.

[27] B. J. Schmitt, W. von Wahl, Decomposition of Solenoidal Fields into Poloidal Fields, Toroidal Fields and the Mean Flow. Applications to the Boussinesq-Equations, Lecture Notes in Math., 1530, Springer, Berlin (1992), 291-305.

[28] S. L. Sobolev, Applications of Functional Analysis in Mathematical Physics, 7, American Mathematical Society, 1963.

[29] S. G. Miklin, An advanced course of Mathematical Physics, North-Holland, Amsterdam London 1970. 